\title{
Valeur alimentaire des noyaux de dattes et effet du taux d'incorporation dans la ration
}

\author{
B Vanabelle, Y Larondelle \\ Université catholique de Louvain, Faculté des Sciences agronomiques, Unité de Biochimie et de Nutrition, Place
} Croix du Sud, 2/8, B 1348 Louvain-la-neuve, Belgique

Des mesures de digestibilité réalisées selon un schéma expérimental en cross-over sur 6 moutons antenais castrés de race Texel pesant en moyenne $43,8 \mathrm{~kg}( \pm 4,57)$ en début d'essai, ont permis de comparer la valeur alimentaire des noyaux de dattes dans trois rations (A, B et C) comportant respectivement 31,$4 ; 51,7$ et $75,8 \%$ de noyaux de dattes ensilés, séchés et broyés (MO $=98,7 \%$, MAT $=6,1 \%, \mathrm{CB}=27,0 \%, \mathrm{MG}=$ $9,2 \%$ de la MS). Ces rations étaient complémentées par du foin de prairie incorporé à raison de 68,$6 ; 38,4$ ou $9,5 \%$ ainsi que par du tourteau de soja utilisé à raison de $0 ; 9,9$ et $14,7 \%$. Les MAT des trois rations n'étaient pas identiques $(8,5 \%, 11,5 \%$ et $12,5 \%$ pour $A, B, C$, respectivement) étant donné l'incorporation de tourteau de soja dans les rations $\mathrm{B}$ et $\mathrm{C}$.

Les coefficients de digestibilité de MO, MAT, CB et MG des noyaux de dattes ont été calculés par différence sur la base des valeurs des tables INRA (1978, Alimentation des ruminants, 552553) pour le tourteau de soja et, pour le foin sur la base des valeurs déterminées expérimentalement sur 3 moutons nourris ad libitum. Les valeurs énergétiques nettes (UFL et UFV) des noyaux de dattes ont été calculées selon les formules de I'INRA (1988, Alimentation des bovins, ovins et caprins) à partir de la valeur de l'énergie métabolisable. Les teneurs en énergie des aliments, des fécès et de l'urine ont été mesurées par calorimétrie directe (Swift et al, 1948, J Anim Sci, 475-485).

\section{Rations}

MS ingérée $(\mathrm{kg} / \mathrm{j})$

MO ingérée $/ \mathrm{kg} \mathrm{P}^{0,75}(\mathrm{~g} / \mathrm{j})$

N.A. (23 g MOD/kg P0.75)

Digestibilité des Noyaux de dattes (\%)

- Matière organique (MO)

- Matières azotées totales (MAT)

- Cellulose brute (CB)

- Matières grasses (MG)

Valeurs énergétiques nettes: UFL/kg MS UFV $/ \mathrm{kg} M S$
Les quantités de MS et de MO volontairement ingérées ainsi que le niveau d'alimentation (NA) atteint augmentent significativement entre la ration $A$ et les rations $B$ et $C$ non significativement différentes. Le niveau d'alimentation et le taux d'incorporation n'influencent pas significativement la digestibilité de $\mathrm{MO}, \mathrm{CB}$ et $M G$. Une tendance à la diminution est cependant observée pour la ration $\mathrm{C}$. La digestibilité des MAT des noyaux de dattes est faible et différente pour les rations $A$ et $C$ (différence probablement due à la méthode de calcul). En effet, une même valeur a été attribuée à la digestibilité des MAT du tourteau de soja bien que les taux d'incorporation soient différents. Les digestibilités des MAT des rations $A$, B et $C$ étant respectivement de $40,5 \%, 55,5 \%$ et $55,6 \%$,il ressort du calcul par différence de la digestibilité des MAT des noyaux de dattes, une sousestimation d'autant plus grande que la part des MAT apportées par les noyaux de dattes est faible. Les valeurs énergétiques nettes des noyaux de dattes, en considérant un même niveau d'alimentation, sont élevées dans les rations $A$ et $B$ ( 1 UFL ou UFV/kg MS), mais plus faibles dans la ration $C(<0,9$ UFL ou UFV $/ \mathrm{kg}$ MS).

En conclusion, la ration B ( $50 \%$ de noyaux) permet d'optimiser la valeur alimentaire des noyaux de dattes en maximisant la valeur énergétique et les quantités volontairement ingérées.

$\begin{array}{ccc}\text { A } & \text { B } & \text { C } \\ 0,974 \mathrm{a} & 1,101 \mathrm{~b} & 1,150 \mathrm{~b} \\ 51,73 \mathrm{a} & 58,73 \mathrm{~b} & 62,29 \mathrm{~b} \\ 1,38 \mathrm{a} & 1,70 \mathrm{~b} & 1,78 \mathrm{~b} \\ & & \\ 64,74 \mathrm{a} & 66,87 \mathrm{a} & 62,40 \mathrm{a} \\ 27,34 \mathrm{a} & 16,55 \mathrm{ab} & 5,26 \mathrm{~b} \\ 66,96 \mathrm{a} & 67,70 \mathrm{a} & 59,82 \mathrm{a} \\ 91,66 \mathrm{a} & 92,83 \mathrm{a} & 88,17 \mathrm{a} \\ 0,99 & 0,99 & 0,88 \\ 0,99 & 1,00 & 0,86\end{array}$

Les valeurs d'une ligne donnée accompagnées d'une même lettre ne diffèrent pas significativement (0.05) 